\section{Corioamnionitis, definición, métodos de diagnóstico y repercusión clínica}

Chorioamnionitis, definition, diagnostic methods and clinical repercussion

Christian David Verdy Martínez Especialista en Ginecología y Obstetricia

Hospital General Guasmo Sur, cdavidvm@hotmail.com,

https://orcid.org/0000-0002-7174-

2509, Guayaquil, Ecuador

Erick Fabian Matute Guerrido Médico general

Hospital Dr. Rafael Rodríguez

Zambrano, matute-

1989@hotmail.com,

https://orcid.org/0000-0002-5705-

7783, Guayaquil, Ecuador

Nadia Dayan Vásquez Vera

Médico general

Hospital Básico Dr. Juan Montalvo

Cornejo, nadiavv7@@hotmail.com,

https://orcid.org/0000-0002-8204-

7480, Guayaquil, Ecuador

Erick Vicente Andrade Palacios

Médico general

Distrito 12D02 Pueblo Viejo-

Urdaneta,

erickvicenteandrade@gmail.com,

https://orcid.org/0000-0001-9872-

3570, Guayaquil, Ecuador

http://www.jah-

journal.com/index.php/jah

Journal of American health

Julio - Diciembre vol. 3. Num. 2 - 2020

Esta obra está bajo una Licencia

Creative Commons

Atribución-NoComercial-

CompartirIgual 4.0 Internacional.

RECIBIDO: 7 DE FEBRERO 2019

ACEPTADO: 21 DE AGOSTO 2019

PUBLICADO: 2 DE JULIO 2020

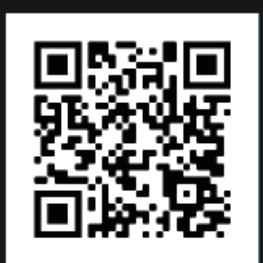

Scan this QR

code with your

smart phone or

mobile device to

read more papers

\section{RESUMEN}

La corioamnionitis es una complicación común del embarazo asociada con resultados adversos maternos, perinatales y a largo plazo significativos. El diagnóstico es problemático y se basa en criterios clínicos y de laboratorio utilizados de manera inconsistente con un valor predictivo pobre. El objetivo de este estudio es examinar el desempeño de los criterios clínicos y paraclínicos para el diagnóstico de corioamnionitis, además de analizar la información actual sobre definición, epidemiología y fisiopatología de la enfermedad. La muestra estuvo conformada por 20 artículos de revisión de la literatura, ensayos y metaanálisis publicados entre enero del 2015 y febrero del 2020. Se utilizó Pubmed, Scielo y Elsevier como motores de búsqueda de la información. El idioma se limitó para artículos en inglés y español.

PALABRAS CLAVE: corioamnionitis, parto pretérmino, ruptura prematura de membranas.

\section{ABSTRACT}

Chorioamnionitis is a common complication of pregnancy associated with significant maternal, perinatal, and long-term adverse outcomes. Diagnosis is problematic and is based on inconsistent clinical and laboratory criteria with poor predictive value. The objective of this study is to examine the performance of clinical and paraclinical criteria for the diagnosis of chorioamnionitis, in addition to analyzing current information on the definition, epidemiology and pathophysiology of the 
disease. The sample consisted of 25 literature review articles, trials and metaanalyzes published between January 2015 and February 2020. Pubmed, Scielo and Elsevier were used as search engines for information. Language was limited for articles in English and Spanish.
KEYWORDS: chorioamnionitis, preterm delivery, premature rupture of membranes.

\section{INTRODUCCIÓN}

La corioamnionitis (CA), inflamación aguda del amnios y el corion, es un marcador de infección intraamniótica, aunque puede ocurrir en ausencia de infección detectable (1). La corioamnionitis histológica, identificada a través del examen patológico de la placenta, puede estar presente en ausencia de corioamnionitis clínica, que se diagnostica principalmente por fiebre materna (2). Tales casos subclínicos representan el 40-90\% de los casos de corioamnionitis histológica, con factores, que incluyen variaciones en las definiciones de corioamnionitis clínica utilizadas en diferentes estudios, lo que contribuye a la variabilidad (3). Además, la infección intraamniótica puede conducir a una respuesta inflamatoria fetal, que se identifica histológicamente por vasculitis fetal, es decir, inflamación de los vasos fetales de la placa coriónica o el cordón umbilical.

Representa el diagnóstico más frecuente en los informes de patología placentaria, y generalmente se considera que representa la presencia de infección intraamniótica o síndrome de infección por líquido amniótico. Sin embargo, la corioamnionitis aguda puede ocurrir con "inflamación intraamniótica estéril", que ocurre en ausencia de microorganismos demostrables, pero puede ser inducida por señales de peligro liberadas bajo condiciones de estrés celular, lesión o muerte. Por lo tanto, la corioamnionitis aguda es evidencia de inflamación intraamniótica y no de infección intraamniótica.

Se estima que del 1-5\% de los fetos mayores de 35 semanas de gestación nacen de madres con corioamnionitis, aunque la incidencia informada en la literatura varía ampliamente según la población estudiada y los criterios de diagnóstico utilizados (1,3). Este síndrome es la contrapartida del síndrome de respuesta inflamatoria sistémica en adultos; sin embargo, en los fetos, es un factor de riesgo para complicaciones a corto y largo plazo (es decir, sepsis neonatal, displasia broncopulmonar, leucomalacia periventricular y parálisis cerebral).

Este artículo revisa la definición, la patogénesis, la clasificación y la estadificación, y la importancia clínica de las lesiones más comunes en la patología placentaria. Además, se analizará las respuestas inflamatorias agudas desplegadas por la madre y el feto en respuesta a estímulos inflamatorios dentro de la cavidad amniótica. 


\section{MATERIALES Y MÉTODOS}

Se realizó una búsqueda exhaustiva en la literatura mediante Pubmed, Scielo y Elsevier. Debido al extenso y diverso tema de la corioamnionitis, la búsqueda se limitó desde el 2014 hasta la fecha en que se realizó la búsqueda en febrero del 2020. Se seleccionaron estudios en idioma inglés y español relacionados con la definición, epidemiología, significado clínico y métodos de diagnóstico de corioamnionitis. Las directrices de las principales sociedades profesionales, metanálisis, ensayos aleatorios y artículos de revisión de la literatura se priorizaron para su revisión. Se utilizaron descriptores de la salud y los medical subject headings para la selección de las palabras clave para la búsqueda de la información.

Los términos de búsqueda para MEDLINE a través de PubMed se muestran a continuación y se modificaron para la terminología de búsqueda de las otras bases de datos: intrauterinas [tw] $\mathrm{O}$ infección uterina [tw] $O$ infecciones uterinas [tw] $O$ infección uterina [tw] $O$ infecciones uterinas [ tw] $\mathrm{O}$ infección intraamniótica [tw] O infecciones intraamnióticas [tw]) Y (Embarazo [malla] O embarazo [tw] O embarazada [tw]). Además, se realizó una búsqueda de literatura gris que identificó los libros de texto de la última edición de uso común en obstetricia y ginecología, agregándose 1 documento al estudio para su revisión. La muestra quedó conformada por un total de 15 artículos científicos de revisión y 5 artículos de análisis de datos.

\section{RESULTADOS}

La corioamnionitis es un término que abarca un amplio espectro de enfermedades durante el embarazo que se caracteriza por inflamación y/o infección de estructuras intrauterinas como la placenta, el corion y el amnios $(1,2,3)$. La presentación clínica de la corioamnionitis puede variar en función de factores clínicos, microbiológicos e histológicos que interactúan y se superponen en diversos grados. Los signos y síntomas dependen de si se encuentra un proceso inflamatorio primario versus un proceso infeccioso (4). La inflamación placentaria a menudo es clínicamente silenciosa y puede indicar el proceso fisiológico normal del parto, un proceso inflamatorio, pero también puede ser un signo de infección subclínica (5). La identificación de una etiología infecciosa, como un cultivo positivo de líquido amniótico, o el desarrollo de hallazgos clínicos, son indicativos de un proceso patológico que puede progresar a una enfermedad materna y neonatal más grave. La distinción de etiología inflamatoria versus infecciosa dentro del espectro de corioamnionitis es, por lo tanto, importante, dadas las profundas diferencias en la morbilidad materna y neonatal posterior.

\section{Definición}

La corioamnionitis o infección intraamniótica es una inflamación aguda de las membranas y el corion de la placenta, generalmente debido a una infección bacteriana polimicrobiana ascendente en el contexto de la ruptura de la membrana (1). Puede ocurrir con membranas 
intactas, especialmente cuando hay asociación con especies de Ureaplasma y Mycoplasma hominis, que se encuentran en el tracto genital inferior de más del $70 \%$ de las mujeres. Solo en raras ocasiones la diseminación hematógena está implicada en corioamnionitis, como ocurre con Listeria monocytogenes $(2,5)$.

El término "corioamnionitis clínica" se refiere a una entidad diagnosticada por la presencia de fiebre $\left(>37.8^{\circ} \mathrm{C}\right)$ y al menos dos de los siguientes criterios: taquicardia materna ( $>100$ latidos por minuto), leucocitosis materna [recuento de glóbulos blancos (GB))> 15,000 células/mm3], sensibilidad uterina, taquicardia fetal (> 160 latidos por minuto) y líquido amniótico (FA) maloliente $(1,2,3,5)$. En general, del 1 al $4 \%$ de todos los nacimientos en los Estados Unidos son complicados por corioamnionitis; sin embargo, la frecuencia de corioamnionitis varía notablemente según los criterios diagnósticos, factores de riesgo específicos y la edad gestacional (4). La corioamnionitis complica hasta 40 a $70 \%$ de los nacimientos prematuros con rotura prematura de membranas o parto espontáneo y 1 a $13 \%$ de los nacimientos a término. El doce por ciento de los partos por cesárea primaria a término implican corioamnionitis clínica, y la indicación más común de cesárea en estos casos es la falta de progreso, generalmente después de la ruptura de la membrana (6).

\section{Manifestaciones clínicas}

Los hallazgos clínicos clave asociados con la corioamnionitis clínica incluyen fiebre, sensibilidad uterina del fondo, taquicardia materna ( $>100 / \mathrm{min}$ ), taquicardia fetal ( $>160 / \mathrm{min}$ ) y líquido amniótico purulento o asqueroso. La fiebre materna es el signo clínico más importante de corioamnionitis (1). La temperatura $>100.4^{\circ} \mathrm{F}$ se considera anormal en el embarazo. Si bien la fiebre de bajo grado aislada $\left(<101^{\circ} \mathrm{F}\right)$ puede ser transitoria en el trabajo de parto, la fiebre $>$ $100.4^{\circ} \mathrm{F}$ persiste más de 1 hora o cualquier fiebre $\geq 101^{\circ} \mathrm{F}$ merece evaluación e intervención apropiada $(5,6)$.

La fiebre está presente en el $95-100 \%$ de los casos de corioamnionitis clínica y generalmente se requiere para el diagnóstico (2). La fiebre en el contexto de la anestesia epidural, particularmente entre las mujeres nulíparas con trabajo de parto prolongado (llamada fiebre epidural) a menudo se encuentra y plantea un atolladero de diagnóstico frente a la corioamnionitis (3). Esto se debe a que i) además de la fiebre, las dos afecciones comparten otros factores de riesgo importantes (baja paridad y trabajo de parto prolongado), ii) la anestesia epidural enmascara los signos de corioamnionitis, como sensibilidad en el fondo, y iii) los medicamentos administrados durante la anestesia epidural pueden inducir a la madre o taquicardia fetal y, por lo tanto, confunden el diagnóstico de corioamnionitis $(5,6)$. Se desconoce el mecanismo exacto de la fiebre epidural, pero se cree que es el resultado del bloqueo simpático epidural de procesos termorreguladores como la sudoración. 
La taquicardia materna (> $100 \mathrm{lpm}$ ) y la taquicardia fetal (> $160 \mathrm{lpm}$ ) ocurren con frecuencia en corioamnionitis, notificándose en $50-80 \%$ y $40-70 \%$ de los casos, respectivamente (7). La taquicardia puede estar presente en ausencia de corioamnionitis y requiere una evaluación cuidadosa para las etiologías alternativas. Los medicamentos como la efedrina, los antihistamínicos y los agonistas beta pueden aumentar la frecuencia cardíaca materna o fetal (8). Sin embargo, la combinación de fiebre materna y taquicardia materna y/o fetal son muy sugestivas de infección intrauterina y deben tratarse en consecuencia. Además de las mediciones objetivas de fiebre materna y taquicardia, otros signos de corioamnionitis son altamente subjetivos.

La sensibilidad del fondo uterino y un olor desagradable del líquido amniótico se informan solo en el $4-25 \%$ de los casos de corioamnionitis $(3,8)$. La sensibilidad del fondo uterino es difícil de interpretar en el contexto del dolor del trabajo de parto y puede enmascararse con analgésicos o confundidos por el dolor asociado con el desprendimiento de la placenta. La purulencia o el mal olor del líquido amniótico tienen más probabilidades de estar presentes con infección severa o prolongada y pueden ser específicos del organismo, pero en cualquier caso los médicos pueden apreciarlo o no.

La corioamnionitis que es subclínica por definición no presenta los signos clínicos anteriores, pero puede manifestarse como trabajo de parto prematuro o, aún más comúnmente, como ruptura prematura de membranas (RPM) pretérmino (5). Además, la RPM a término (ruptura de la membrana $\geq 37$ semanas de gestación, pero antes del inicio de las contracciones uterinas), que ocurre en el $8 \%$ o menos de los nacimientos a término, se asocia con un mayor riesgo de corioamnionitis $(7,8)$.

\section{Patogenia}

Se requieren estímulos quimiotácticos para que los neutrófilos migren al tejido. Estos estímulos son proporcionados por quimiocinas de neutrófilos (IL-8, también conocido como péptido activador de neutrófilos, y CXCL6 - proteína quimiotáctica de granulocitos) (4). La inflamación intraamniótica debida a microorganismos o "señales de peligro" puede dar lugar a la producción de las siguientes quimiocinas: IL-8, citocina inhibidora de macrófagos, MCP, MCP2, MCP-3, MIP-1 $\alpha$, CXCL6, CXCL10, CXCL13, ENA-78, RANTES y GRO- $\alpha$. Por lo tanto, las concentraciones de quimiocinas en el líquido amniótico son elevadas y establecen un gradiente quimiotáctico que favorece la migración de neutrófilos $(5,6)$. En ausencia de microorganismos, las señales de peligro liberadas por las células en condiciones de estrés o muerte celular pueden inducir inflamación intraamniótica (inflamación estéril) (8). El diagnóstico de esta afección es de exclusión y requiere un examen del líquido amniótico con técnicas de cultivo y microbiológicas moleculares. 


\section{Métodos de diagnóstico}

\section{Laboratorio}

La leucocitosis materna es el hallazgo de laboratorio más utilizado en el diagnóstico de corioamnionitis clínica. Se utilizó un recuento de glóbulos blancos (WBC) mayor o igual a $15,000 / \mathrm{mm}^{3}$ como punto de corte para este criterio. Debe considerarse que la leucocitosis materna es relativamente inespecífica y puede ser inducida por varios factores, incluidos los corticosteroides prenatales (1). Los corticosteroides prenatales son especialmente pertinentes ya que a menudo se administran a pacientes que también tienen un alto riesgo de desarrollar corioamnionitis, como aquellos con trabajo de parto prematuro y ruptura prematura de membranas. Existen otras pruebas de laboratorio como la proteína $C$ reactiva, interleucina-6, molécula de adhesión intracelular soluble (sICAM), procalcitonina, proteína de unión a lipopolisacáridos y la metaloproteinasa-8, sin embargo, son de valor limitado clínicamente y a menudo se usan solo en investigación ajustes (9).

\section{Hallazgos histológicos}

La asociación entre los hallazgos histológicos de corioamnionitis en la placenta y la infección está bien establecida. Se ha encontrado que los hallazgos histológicos positivos son más sensibles que la corioamnionitis clínica confirmada mediante cultivo de líquido amniótico (2). Además, la corioamnionitis histológica en término, los embarazos de bajo riesgo a menudo se asocian con inflamación placentaria en lugar de infección placentaria. El diagnóstico de corioamnionitis histológica se realiza de forma retrospectiva después del parto. Los criterios de diagnóstico se basan en la etapa y el grado de invasión de leucocitos polimorfonucleares maternos por campo de alta potencia en la placa placentaria y en las membranas, desde el corion hasta el amnios en dirección amniotrópica (8). Existen varios criterios de clasificación y clasificación que se han utilizado en la literatura con respecto a los hallazgos patológicos de corioamnionitis dentro de la placenta y las membranas e incluyen los criterios Redline, Salafia y Blanc (9).

\section{Hallazgos microbiológicos}

Si bien numerosos estudios han demostrado la correlación entre el cultivo positivo de líquido amniótico y la corioamnionitis, también se pueden encontrar cultivos positivos de líquido en infecciones subclínicas. Del mismo modo, los resultados positivos del cultivo de bacterias patógenas de los hisopos entre las capas de la membrana placentaria, el corion y el amnios, se correlacionan con la infección intraamniótica (3). La mayoría de las infecciones intraamnióticas 
son de origen ascendente desde el tracto genital y son polimicrobianas, con organismos tanto anaerobios como aerobios aislados. En un estudio, se encontró que las mujeres con infección aguda intraamniótica tenían tasas más altas de aislamientos de alta virulencia en comparación con los controles (10). Estos incluyeron estreptococos del grupo B, estreptococos hemolíticos $\alpha$, Escherichia coli, Clostridium spp, Bacteroides spp, entre otros. Otras rutas de infección descritas incluyen la diseminación hematógena de bacterias como Listeria monocytogenes, estreptococos del grupo A y Campylobacter spp (11).

El riesgo de infección intraamniótica iatrogénica por la terapia fetal invasiva o los procedimientos de diagnóstico prenatal es bajo si se toman las precauciones adecuadas. Otras pruebas sobre el líquido amniótico, que incluyen el nivel de glucosa, la actividad del lactato deshidrogenasa, el recuento de glóbulos blancos y la tinción de Gram, son menos confiables para identificar y confirmar la corioamnionitis. Las pruebas de amplificación nuclear, como la reacción en cadena de la polimerasa (PCR) para la detección de infección intraamniótica, se utilizan principalmente con fines de investigación (11).

\section{Repercusión clínica}

\section{Sepsis neonatal}

Los neonatos nacidos después de una CA tienen un mayor riesgo de infección neonatal. Los organismos que infectan las membranas coriónicas están muy cerca del feto y pueden causar infección fetal como consecuencia natural de esta proximidad. Los organismos que con más frecuencia causan la aparición de sepsis neonatal temprana son los que son también causas frecuentes de CA: Escherichia coli y el grupo B Streptococcus. La exposición a CA parece conferir un riesgo de sepsis de inicio temprano de 1-3\%, 10 veces mayor que el riesgo general de sepsis de inicio temprano $(1,11)$.

\section{Enfermedad cerebral}

La CA se asocia con una mayor incidencia de lesiones neurológicas tempranas, que incluyen hemorragia intraventricular severa (HIV) y leucomalacia periventricular (LPV), que son causas establecidas de deterioro del desarrollo neurológico en bebés prematuros. Incluso cuando no se documenta un diagnóstico perinatal de HIV o LPV, la CA parece estar asociado con un mayor riesgo de parálisis cerebral (11). 


\section{Enfermedad pulmonar}

La relación de corioamnionitis y la morbilidad pulmonar neonatal es conflictiva. Varios estudios han evaluado el riesgo de síndrome de dificultad respiratoria (SDR) y displasia broncopulmonar (DBP) después de la exposición a CA (11).

\section{Displasia broncopulmonar (DBP)}

La relación entre DBP y CA también es conflictiva. La razón para un mayor riesgo de DBP después de $C A$ es que la exposición intrauterina a la inflamación puede causar un desarrollo pulmonar posterior que sea anormal y conduzca a DBP (11).

\section{DISCUSIÓN}

El diagnóstico clínico de corioamnionitis es una indicación para la administración de antimicrobianos. Los resultados de las pruebas de laboratorio pueden ayudar a descartar el diagnóstico de corioamnionitis, especialmente cuando los signos y síntomas clínicos son equívocos. La leucocitosis materna (definida de manera diversa como WBC $>12,000 / \mathrm{mm} 3$ o> $15,000 / \mathrm{mm} 3$ ) o la presencia de un desplazamiento a la izquierda o una bandemia (> 9\%) a menudo respaldan el diagnóstico de corioamnionitis (12). Se informa leucocitosis en aproximadamente el $70-90 \%$ de los casos de corioamnionitis clínica. Sin embargo, la leucocitosis aislada en ausencia de otros signos o síntomas tiene un valor limitado, ya que puede ser inducida por varias otras afecciones, incluido el trabajo de parto y el uso de esteroides (13). Por lo tanto, el monitoreo rutinario del conteo de glóbulos blancos en mujeres de alto riesgo (ruptura prematura de la membrana pretérmino) en ausencia de signos clínicos de corioamnionitis no es útil.

Otros parámetros de laboratorio que incluyen altos niveles de proteína $C$ reactiva $(P C R)$, proteína de unión a lipopolisacárido, molécula de adhesión intercelular soluble-1 e interleucina- 6 se han asociado con un mayor riesgo de corioamnionitis en el contexto de RMP o parto pretérmino (13). Sin embargo, su utilidad para el diagnóstico o la predicción de corioamnionitis como parte de la práctica clínica habitual no está establecida.

Las pruebas de líquido amniótico, generalmente obtenidas por amniocentesis, se han utilizado para el diagnóstico de corioamnionitis (2). El cultivo de líquido amniótico es la prueba más confiable, pero es de utilidad limitada ya que los resultados del cultivo pueden no estar disponibles por hasta 3 días. Además, debido a la naturaleza invasiva del procedimiento, la amniocentesis no se realiza en la mayoría de los casos, que ocurren durante el trabajo de parto (8). Algunos médicos usan la amniocentesis para confirmar la sospecha clínica de corioamnionitis con el fin de determinar si el parto pretérmino está justificado (evitando así la 
"prematuridad iatrogénica") (10). La amniocentesis también se usa en algunos centros para identificar corioamnionitis subclínica en mujeres con trabajo de parto prematuro espontáneo y ruptura de la membrana prematura al inicio edades gestacionales. Sin embargo, el valor de esta práctica ha sido cuestionado recientemente.

El parto pretérmino es la consecuencia más demostrada de la CA. Un estudio observacional prospectivo temprano de 2774 parejas de madres e infantes encontró que hasta el $25 \%$ de los nacimientos prematuros son atribuibles a CA (13). Esto se confirmó más tarde en un estudio de casos y controles que demostró que los neonatos pretérmino tienen probabilidades significativamente mayores de aislamiento bacteriano y hallazgos histológicos de CA. Un estudio prospectivo reciente de 871 embarazos descubrió que, entre las personas con CA histológica, el parto prematuro ocurrió casi el doble de veces que las personas sin CA (14). Cuando el Ureaplasma urealyticum se detectó después de la ruptura prematura de membranas pretérmino de 154 embarazos, la mediana de edad gestacional al momento del parto fue de 4 semanas menos que cuando no se detectó. De 50 mujeres que se presentaron en trabajo de parto pretérmino con membranas intactas, los niveles intraamnióticos elevados de las citocinas proinflamatorias IL-6, interleucina-1, prostaglandina E2 y factor de necrosis tumoral alfa se asociaron con la progresión al parto prematuro (7).

Los neonatos pretérmino son particularmente susceptibles a la sepsis después de la CA. Un estudio de casos y controles encontró que los lactantes con muy bajo peso al nacer expuestos a CA tenían probabilidades significativamente mayores de sepsis de inicio temprano, $\mathrm{OR}=4.7$ $(1.4,15.9), p=0.015$ (15). Para los lactantes < 32 semanas de edad gestacional, 145 lactantes expuestos a CA histológica desarrollaron criterios clínicos consistentes con el síndrome de sepsis significativamente más frecuente que 136 lactantes $\sin C A, 39 \%$ vs $24 \%(p=0.007)(16)$.

Los recién nacidos a término también corren el riesgo de infección neonatal después de la corioamnionitis. De 5144 recién nacidos a término expuestos a CA, el 1.3\% desarrolló sepsis neonatal comprobada por cultivo, $O R=2.9(2.1,4.1)(17)$. Un análisis de 3094 nacimientos encontró que un diagnóstico clínico de CA se asoció de forma independiente con mayores probabilidades de sepsis neonatal de inicio temprano, incluso con el ajuste de la gravedad inicial de la enfermedad y las características basales, $O R=5.54(2.87,10.69), p<0.001$ (10).

La funisitis parece conferir un mayor riesgo de sepsis. Cuando se consideraron 315 nacimientos pretérminos, el $12 \%$ de los neonatos con funisitis tuvieron un hemocultivo positivo dentro de las 72 horas posteriores al parto en comparación con el $1 \%$ de los neonatos sin funisitis, OR = $7.2(1.8,29.0)$. Un estudio observacional prospectivo de 231 neonatos encontró que la sepsis de inicio temprano ocurrió en el $18 \%$ de los neonatos con funisitis en comparación con el $4 \%$ de los neonatos sin funisitis, $p=0.002$ (3). 
Un estudio multicéntrico de hospitales de la Red Neonatal Canadiense encontró que la CA aumentó las probabilidades de HIV grave incluso después de ajustar la gravedad de la enfermedad, $\mathrm{OR}=1.62(1.17,2.24)$. La presencia de células inflamatorias mononucleares en las membranas fetales se ha asociado con un aumento de la HIV grave incluso después de controlar la edad gestacional, $\mathrm{OR}=8.9(2.1,37.9)$. Un estudio de 1367 lactantes MBPN encontró que la exposición a CA clínica aumentó las probabilidades tanto de HIV, OR = $2.8(1.6,4.8)$ como de LPV, OR = $3.4(1.6,7.3)(18)$.

La relación entre la corioamnionitis y el mal resultado del desarrollo neurológico no se ha demostrado en todos los estudios. Para 39 recién nacidos de $<32$ semanas de gestación que estuvieron expuestos a CA en comparación con 33 recién nacidos de control, no hubo diferencias entre los grupos en los índices de desarrollo mental y psicomotor (18). De manera similar, un estudio de casos y controles no encontró diferencias en el rendimiento en las Escalas de Bayley de desarrollo Infantil entre los 71 niños expuestos y los 259 niños no expuestos a CA a los 7 meses de edad corregida (19).

Sobre la displasia broncopulmonar (DBP), un metaanálisis que incluye estudios de 1994-2009 encontró que la $\mathrm{AC}$ histológica, pero no clínica, se asoció con un aumento de las probabilidades de $\mathrm{DBP}, \mathrm{OR}=2.19(1.76,2.72)$. Los datos que no respaldan una asociación entre CA y BPD incluyen una evaluación histológica de 446 placentas que no lograron encontrar una diferencia en la incidencia de DBP para aquellos con y sin infiltración celular inflamatoria (20).

\section{CONCLUSIONES}

La corioamnionitis aguda, la funisitis aguda y la vasculitis coriónica son lesiones inflamatorias agudas con importancia clínica importante a corto y largo plazo. Se han realizado progresos sustanciales en la comprensión de los mecanismos responsables de la inflamación materna y fetal en el contexto de la infección. Se desconocen las causas de la inflamación intraamniótica estéril y representan importantes desafíos clínicos y científicos.

Representa un problema común, especialmente entre los partos pretérmino. El diagnóstico se realiza con mayor precisión a partir de muestras patológicas. Para la ruptura prematura de membranas pretérmino, se deben usar antibióticos para reducir el riesgo de parto pretérmino y sus complicaciones posteriores. Se recomienda la administración de antibióticos a los recién nacidos después de una corioamnionitis. Los estudios futuros deberían explorar mejores modalidades de diagnóstico y tratamiento.

Una estrategia de manejo que consiste en la observación cercana de los recién nacidos a término y prematuros expuestos a sospecha de infección intrauterina es preferible a la terapia antimicrobiana empírica. Se necesitarán grandes estudios epidemiológicos prospectivos para 
determinar el impacto de estas nuevas pautas de práctica en los resultados de los neonatos expuestos a la infección y/o inflamación intrauterina. La precisión mejorada en el diagnóstico clínico de la infección intrauterina debería mejorar tanto la calidad como la reproducibilidad de los datos generados en futuros estudios.

\section{REFERENCIAS}

1. Couto D. Corioamnionitis y parto pretérmino: resultados perinatales en un trienio. MEDISAN. Vol.20(12): 2489. 2016.

2. Hurtado F. Criterios actuales en el diagnóstico y manejo de la Corioamnionitis. Rev. Latin. Perinat. Vol. 21 (1): 11-17. 2018.

3. Kachikis et al. Chorioamnionitis: Case definition \& guidelines for data collection, analysis, and presentation of immunization safety data. Vaccine. Vol. 37(52):7610-7622. doi:10.1016/j.vaccine.2019.05.030. 2019.

4. Kim et al. Acute chorioamnionitis and funisitis: definition, pathologic features, and clinical significance. Am J Obstet Gynecol. Vol. 213(4 Suppl):S29-S52. doi:10.1016/j.ajog.2015.08.040. 2015.

5. Peng C. Intrauterine inflammation, infection, or both (Triple I): A new concept for chorioamnionitis. Pediatr Neonatol. Vol. 59(3):231-237. doi:10.1016/j.pedneo.2017.09.001. 2018.

6. Fukami et al. Histologic chorioamnionitis prevalence in patients with premature rupture membranes. Clin Exp Obstet Gynecol. Vol. 44(2):236-238. 2017.

7. DeNoble A. Chorioamnionitis and Infectious Complications after Vaginal Delivery. Am J Perinatol. Vol. 36(14):1437-1441. doi:10.1055/s-0039-1692718. 2019.

8. Hoffman M. Chorioamnionitis: a reason to deliver but not perform a caesarean. BJOG. Vol. 126(6):728. doi:10.1111/1471-0528.15602. 2019.

9. Fowler J. Chorioamnionitis. In: StatPearls [Internet]. Treasure Island (FL): StatPearls Publishing; Available from: https://www.ncbi.nlm.nih.gov/books/NBK532251/. 2020.

10. Venkatesh K. Association of chorioamnionitis and its duration with adverse maternal outcomes by mode of delivery: a cohort study. BJOG. Vol. 126(6):719-727. doi:10.1111/1471-0528.15565. 2019.

11. Ericson J. Chorioamnionitis: implications for the neonate. Clin Perinatol. Vol. 42(1):155-ix. doi:10.1016/j.clp.2014.10.011. 2015.

12. Cappelletti M. Immunobiology of Acute Chorioamnionitis. Front Immunol. Vol. 11:649.cdoi:10.3389/fimmu.2020.00649. 2020. 
13. Daifotis et al. Risk Factors for Postpartum Maternal Infection Following Spontaneous Vaginal Delivery Complicated by Chorioamnionitis. AJP Rep. Vol. 10(2):e159-e164. doi:10.1055/s-00401709983. 2020.

14. Bastek J. Prenatal inflammation is associated with adverse neonatal outcomes. American journal of obstetrics and gynecology. 2014 May;210(5):450 e451-410. 2014.

15. Ocheke et al. Maternal and neonatal outcomes of histological chorioamnionitis. J West Afr Coll Surg. Vol. 6(3):1-14. 2016. 